\title{
Optimization of the nonlinear optical response in chiral media
}

\author{
V. Ostroverkhov, O. Ostroverkhova, R.G. Petschek, and K.D. Singer \\ Case Western Reserve University, Cleveland, OH 44106 \\ L. Sukhomlinova and R.J. Twieg \\ Kent State University, Kent, OH 44242
}

\begin{abstract}
This paper describes the development and optimization of chiral, non-polar media with large second-order nonlinear optical responses. We employ molecular engineering, quantum-mechanical sum-over-states theory, and measurements of molecular hyperpolarizability by means of Kleinman-disallowed hyper-Rayleigh scattering in order to understand molecular properties. Then we analyze the appropriate arrangement of the chromophores that produce an optimum axial nonlinear optical medium. Chromophores with large Kleinman disallowed traceless symmetric second rank tensor hyper-polarizabilities $\beta$ can be aligned so as to result in large susceptibilities, $\chi^{(2)}$, in structures that lack polar order. We found that L-shaped chromophores with $C_{2 \mathrm{v}}$ or similar symmetry are good candidates for these materials as they can exhibit large second-rank components of the hyperpolarizability tensor. A wide variety of techniques can be used to fabricate bulk materials belonging to the chiral non-polar symmetry groups, $D_{\infty}$ and $D_{2}$. The microscopic chromophore alignment schemes that optimize the NLO response in such materials are deduced from general symmetry consideration for both molecules and bulk.
\end{abstract}

\section{INTRODUCTION}

Second-order nonlinear optics requires a noncentrosymmetric material. Consequently, much effort has gone into the development of polar materials that exploit the dipolar (vector) component of the molecular hyperpolarizability based on a push-pull conjugated model. ${ }^{1}$ However, it has been recognized for a long time that even isotropic fluids containing chiral molecules can exhibit second order nonlinear optical effects. ${ }^{2}$ Second harmonic generation is not observed in such isotropic chiral media, but it may be observed in chiral materials that are at least uniaxially aligned. Since dipoles have low entropy and generally also lower energy when aligned antiparallel, polar macroscopic materials are generally difficult to align and maintain alignment. In contrast the nonpolar axial alignment results in many materials from simple steric interactions, or by stretching of polymers. Many such media lacking full rotation symmetry or mirror planes will have allowed components of the first hyperpolarizability. In chiral media, in which the molecular structure does not allow for any mirror symmetries, together with uniaxial $\left(D_{\infty}\right)$ or biaxial $\left(D_{2}\right)$ alignment are consistent with second order nonlinear polarizabilities, including ones such as second harmonic generation and the electro-optical effect. Such materials can be easily fabricated by aligning of chromophores in media that are expected to have relatively little light scattering. Because of this and intrinsic interest in chirality in general, there has been a recent increase of activity involving the macroscopic nonlinear optical properties of chiral materials. ${ }^{3}$ However, in contrast little has appeared discussing the molecular aspects necessary for obtaining a large nonlinear response in chiral media. ${ }^{4,5}$

We have recently described a scheme to optimize molecular properties of multidimensional NLO chromophores for use in chiral nonlinear materials using molecular engineering, and including sum-over-states quantum-mechanical calculations and measurements of the rotational invariants of molecular hyperpolarizability $b$ by means of Kleinman-disallowed hyper-Rayleigh scattering. ${ }^{6}$ Alternative nonpolar methods of alignment exploit the tensorial nature of the molecular hyperpolarizability b. Generally, the second harmonic hyperpolarizability tensor can be decomposed into four components, two of which transform under three-dimensional rotations as traceless symmetric second- and third-rank tensors. These components of higher rank are responsible for macroscopic second-order nonlinear properties of materials with non-polar order. Optimization of the third-rank (or octupolar) hyperpolarizability has been addressed previously (e.g. Ref. 7), while utilization of the second-rank component, which exists only in species with Kleinman symmetry (full index permutation) breaking is addressed in this paper.

In order to have Kleinman-disallowed hyperpolarizabilities a chromophore must have electrons that move in at least two dimensions. The weight of different irreducible components can be measured by means of Kleinman-disallowed hyperRayleigh scattering (KD-HRS) ${ }^{8,9}$ We have examined a promising class of molecules, namely $\Lambda$-shaped $\pi$-conjugated chromophores that consist of two electron donors (or acceptors) at the ends connected to one acceptor (or donor) at the $\Lambda$ 's vertex. 
In addition, Crystal Violet, which may have a symmetry similar to $D_{3}$ is also interesting. Both have shown strong non-vector components of the hyperpolarizability tensor.

Once the chromophore is optimized for the second-rank tensor component, the next step is to properly embed it into a chiral axial medium. The symmetries $D_{\infty}$ and $D_{2}$ are important cases for nonpolar axial alignment, as we believe that these are easy to fabricate and occur naturally in liquid crystals, for example. Given a chromophore that has a Kleinman-disallowed irreducible hyperpolarizability component, the criteria for axial alignment that efficiently utilize the molecular nonlinearity can be enumerated. We note that chirality of the bulk does not necessarily require chirality of the chromophores so that we will discuss how achiral (but Kleinman-asymmetric) molecules can be arranged in a chiral fashion that will result in an efficient NLO medium.

In further sections of this paper we discuss in detail all the steps of the chiral NLO medium optimization and present recent results of Kleinman-disallowed HRS for a number of $L$-shaped molecules as chromophores for chiral nonpolar NLO media.

\section{CHROMOPHORE CHARACTERIZATION: KLEINMAN DISALLOWED CASE}

\subsection{Irreducible approach}

To begin to understand how to optimize the molecular properties that can be exploited in these chiral media, we express the molecular hyperpolarizability in terms of the irreducible representation of the rotation group. The hyperpolarizability is a rank-3 tensor that transforms as the product of three rank-1 vectors. This can be reduced through the formalism of the addition of angular momenta. ${ }^{10}$ In the most general case of parametric light scattering, the hyperpolarizability, $\beta_{\text {pls }}$, transforms as follows:

$$
\begin{aligned}
\mathrm{b}_{\mathrm{pls}} & \sim 1 \mathrm{f} 1 \mathrm{f} 1 \\
& \sim 0 \approx(1 \approx 1 \approx 1) \approx(2 \approx 2) \approx 3
\end{aligned}
$$

Eq. (1) shows that $\beta$ consists of 1 antisymmetric pseudo-scalar $(\mathrm{L}=0), 1$ fully symmetric rank-3 tensor ( $\mathrm{L}=3$ ), 3 vectors $(\mathrm{L}=1)$ and 2 pseudo-tensors $(\mathrm{L}=2)$. One of the rank-1 objects is fully symmetric, the remaining two of rank-1 and rank-2 components transform as the mixed representation of the permutation group of three objects. Second harmonic hyperpolarizability is a third-rank tensor symmetric under permutation of the last two indices.

$$
\begin{aligned}
\mathrm{b}_{\mathrm{SHG}} & \sim 1 \mathrm{f}(1 \mathrm{f} 1)_{\mathrm{sym}} \\
& \sim 1 \mathrm{f}(0 \approx 2) \\
& \sim 1 \approx 1 \approx 2 \approx 3
\end{aligned}
$$

Group theory provides a mechanism for decomposing this into a sum of four irreducible parts whose components do not mix under three-dimensional rotation due to the fact that they carry different permutation symmetry. In Cartesian coordinates, the decomposition can be written as follows:

$$
b_{i j k}=b_{i j k}^{(3 s)}+b_{i j k}^{(2 m)}+b_{i j k}^{(1 s)}+b_{i j k}^{(1 m)}
$$

Two components ( $1 s$ and $1 m$ ) transform as vectors, $2 m$ - as a second-rank traceless symmetric pseudo-tensor, and $3 s$ - as a third-rank traceless symmetric tensor. The indices $s$ and $m$ denote the parts that are fully symmetric $(s)$ and those that have mixed $(m)$ symmetry under permutations. While there are a number of approaches to extracting rotationally irreducible components, in Cartesian representation this can be done by contracting the tensor $b$ with the fully symmetric Kroneker $d$ tensor and the fully antisymmetric Levi-Civita tensor e The components of lower rank are appropriately embedded in rank-3 tensor form. components:

In the fully symmetric case of Kleinman (full permutation) symmetry, ${ }^{11}$ the hyperpolarizability consists of just two

$$
\mathrm{b}_{\text {sym }} \sim 1 \approx 3
$$

Each irreducible component of hyperpolarizability tensor in the decomposition (3) has a scalar invariant associated with it, which can serve as a natural measure of the component's contribution to the total b. Since there are two components of the same rank in the decomposition (Eq. (3)) ( $1 \mathrm{~s}$ and $1 \mathrm{~m}$ ), there is also a complex-valued invariant defined as a dot product between the two vectors so that there are six (real) scalar rotational invariants that can be extracted from the hyperpolarizability tensor. These invariants can be measured in a specially designed hyper-Rayleigh scattering experiment, making it a powerful tool for characterizing chromophores for chiral NLO. 


\subsection{Quantum-mechanical sum analysis}

The method of optimizing molecular hyperpolarizabilities and the $L=2$ component of interest here can be understood by considering the quantum mechanical expression for the parts that transform like various irreducible representations of the rotation group, that is, like various traceless symmetric tensors. The expression for $\beta$ in terms of microscopic parameters of the molecule can be written down as a perturbative solution to the appropriate Schrödinger equation: ${ }^{12}$

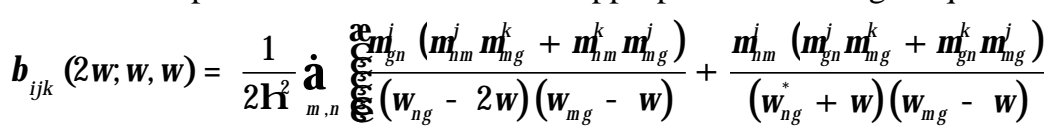

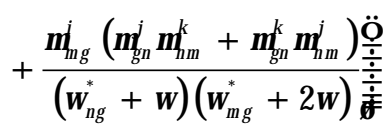

Eq. (4) is a simplified form for the special case of second harmonic generation and the symmetry with respect to last two indices permutation is intrinsic. The ground state is denoted by $g$ and $n$ and $m$ run over all available quantum states. Di ssipation is taken into account by introducing complex-valued transition frequencies $w_{n g} \int w_{n g}^{0}-i G_{n} / 2$ with $w_{n g}^{0}$ being a real transition frequency and $G_{n}$, the dissipation, being inversely proportional to the relaxation time. We can specialize this to the various representations of the rotation group, yielding,

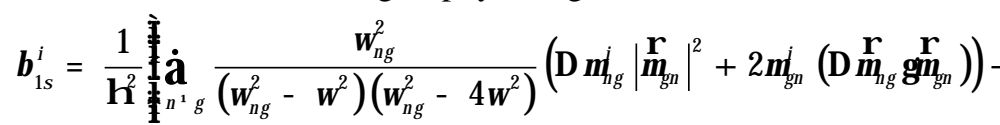

$$
\begin{aligned}
& -\underset{m \pi n g}{\hat{A}} \hat{A} \frac{\dot{z} w^{4}\left(w_{m g}^{2}-4 w_{m g} w_{n g}+w_{n g}^{2}\right)+w^{2} w_{m g} w_{n g}\left(3 w_{m g}^{2}-w_{m g} w_{n g}+3 w_{n g}^{2}\right)-w_{m g}^{3} w_{n g}^{3}}{\left(w_{n g}^{2}-w^{2}\right)\left(w_{n g}^{2}-4 w^{2}\right)\left(w_{m g}^{2}-w^{2}\right)\left(w_{m g}^{2}-4 w^{2}\right)} ¥
\end{aligned}
$$

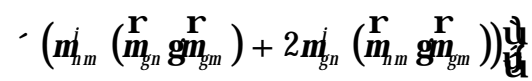

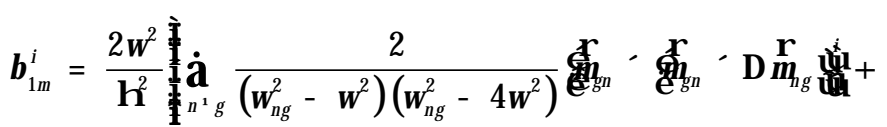

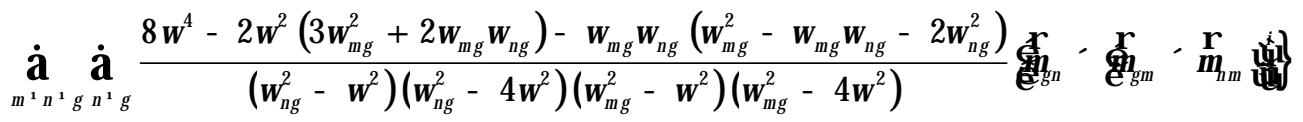

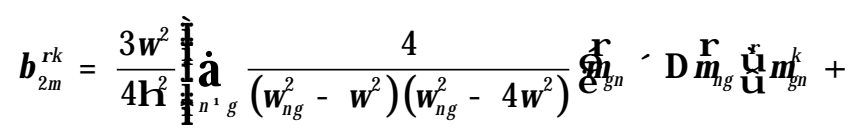

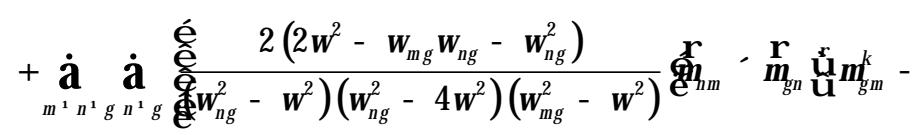

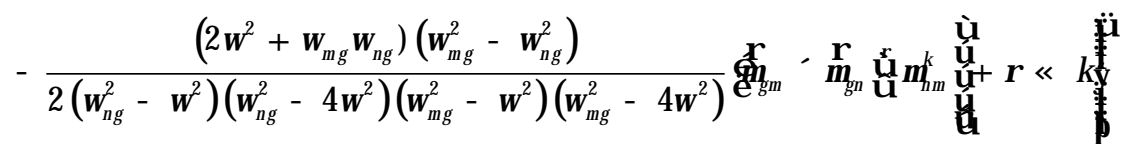

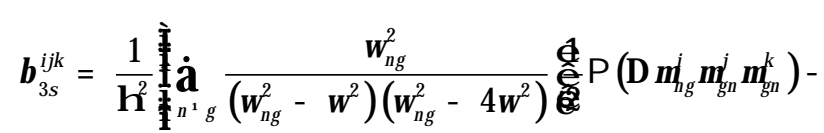

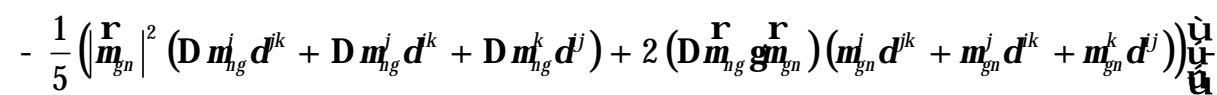

$$
\begin{aligned}
& -\hat{m}_{n \pi g} \hat{A} \frac{2 w^{4}\left(w_{m g}^{2}-4 w_{m g} w_{n g}+w_{n g}^{2}\right)+w^{2} w_{m g} w_{n g}\left(3 w_{m g}^{2}-w_{m g} w_{n g}+3 w_{n g}^{2}\right)-w_{m g}^{3} w_{n g}^{3}}{\left(w_{n g}^{2}-w^{2}\right)\left(w_{n g}^{2}-4 w^{2}\right)\left(w_{m g}^{2}-w^{2}\right)\left(w_{m g}^{2}-4 w^{2}\right)} ¥
\end{aligned}
$$

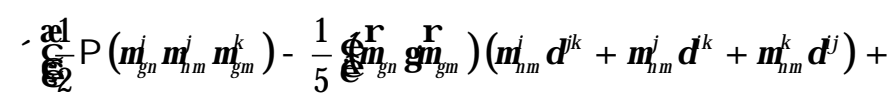

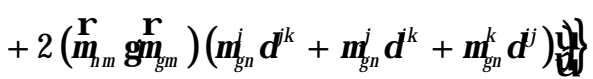


where $D \dot{m}_{\mathrm{ng}}=\dot{m}_{\mathrm{nn}}-\dot{m}_{\mathrm{gg}}$ is the change in the dipole moment and $\mathrm{P}$ is the permutation operator which interchanges the indices $\mathrm{i}, \mathrm{j}$, and $\mathrm{k}$.

The first term in each case contains the two-level contributions, which are well-known to give good de scriptions of the nonlinear optical response in many quasi-one-dimensional charge transfer molecules. ${ }^{13}$ In general, a multilevel model must be applied, and so this general case will be considered first. Of particular interest is the appearance of both dot and cross products in the various terms. For the $1 s$ components, the dot product appears throughout, and thus the largest components are obtained if the various moments for important states are parallel. This feature is well-known in optimizing quasione-dimensional push-pull molecules for polar nonlinear optics. The $3 \mathrm{~s}$ component involves differences including various dot products and, moreover, there are advantages to having more than one polarization of light relative to the molecule active in the non-linear optical behavior. Moreover, there are sum rules involving the transition matrix elements in each direction so that $\beta$ must have components in more than one direction, and there are device structures in which this can be exploited. Thus both multidimensional and linear molecules can have large and useful $3 \mathrm{~s}$ hyperpolarizabilities. We see, however, that cross products appear in the two Kleinman disallowed contributions, the $1 m$ and $2 m$ components, which implies that mult idimensional molecules are a requirement. It is interesting to note that the $1 m$ component contains the cross product in the two-level terms, and the dot product in the others so that orthogonal moments favor the two-level terms, while parallel $\mathrm{m}$ oments favor the others. For the $2 m$ component of primary interest here, we see that orthogonal moments are always favored. Considering a single electronic state contributing in Kleinman-disallowed part of b, one can write the second-rank tensor part as follows:

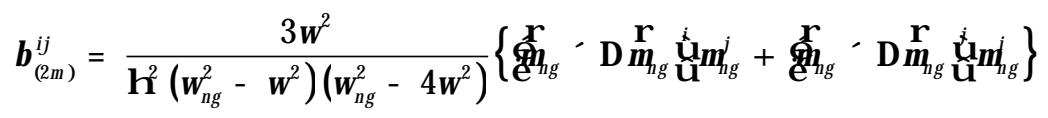

Even though the standard two-level model is not adequate for the description of the molecular response of two- and three-dimensional molecules, our analysis suggests that the hyperpolarizability components of interest to us in $\Lambda$-shaped (or quasi- $\Lambda$-shaped) molecules consisting of two donors and an acceptor (or vice versa) can be described by Eq. (6) in many cases, provided that the states with the correct symmetries are included in the sum. ${ }^{6}$ For understanding the low-lying ele ctronic states, $\Lambda$-shaped molecules that have a single conjugated region consisting of an apex and two identical "feet" can o ften be treated as having $C_{2 v}$ or $C_{2}$ symmetry. The electronic states of these molecules can be either symmetric ( $A$-type) or antisymmetric ( $B$-type) functions with respect to the $180^{\circ}$ rotation. This results in two distinct possibilities for the transition dipole moment: it must be either parallel (for an $A$ state) or perpendicular (for a $B$ state) to the molecular rotation axis, which corresponds to its dipole. Thus, according to Eq. (6), only the $B$-states will contribute to $b_{i j k}^{(2)}$ and, in fact, have optimal g eometry since the $B$-state transition moment is orthogonal to the symmetry axis. ${ }^{6,14}$ To maximize the nonlinear optical $\mathrm{r}$ esponse in chiral media composed of any $\Lambda$-shaped molecules, the lowest-lying state should have substantial $B$-character. Semiempirical calculations for several $\Lambda$-shaped molecules indicate that some molecules of this type indeed do have quite strongly absorbing low-lying states with the appropriate symmetry. ${ }^{15}$ In addition, Eq. (6) indicates that the molecular $\mathrm{r}$ esponse will be enhanced in the anomalous dispersion regime, e.g. for second harmonic generation when the fundamental laser frequency is lower energy but the second harmonic is higher in energy than the energy of the relevant ( $B$ type) quantum state.
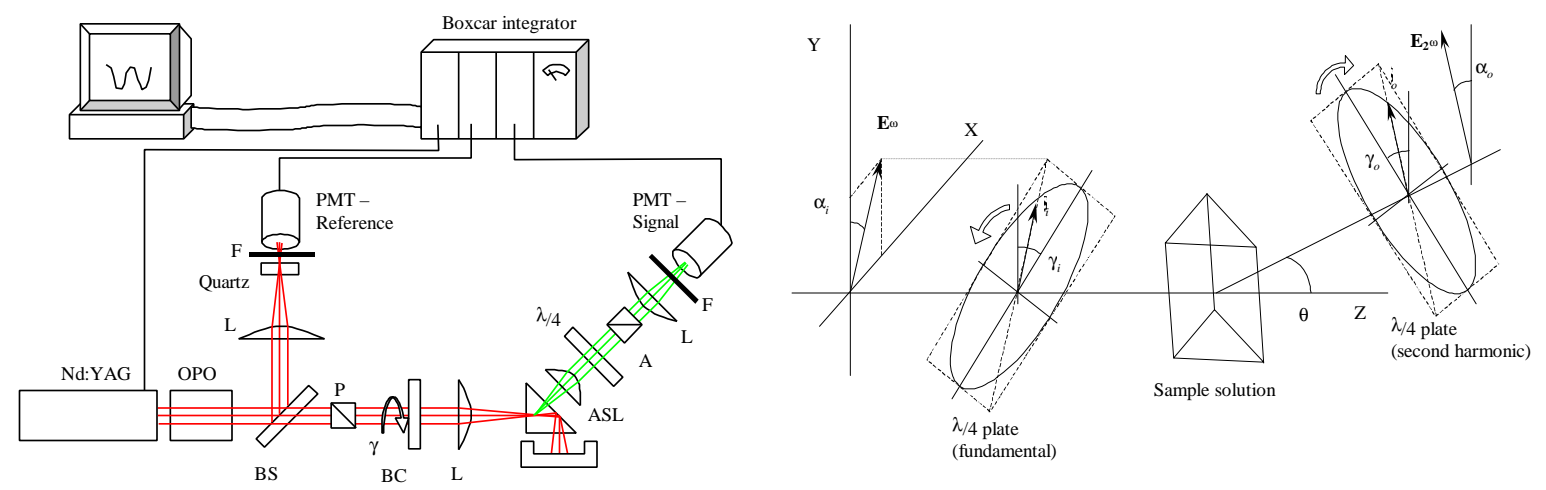

Figure 1. Kleinman-disallowed hyper-Rayleigh scattering: (a) experimental setup ( $\mathrm{P}$ - input polarizer, BS - beamsplitter, L - lenses, $\mathrm{BC}$ - Berek compensator, ASL - aspherical lens, $\lambda / 4$ - quarter-wave plate, A - output polarizer, PMT - photomultiplier tubes, $\mathrm{F}-$ filters); (b) experiment geometry with arbitrary ellipticity of incident and detected light 
In contradistinction the (two level) contributions of a single quantum state (whether $A$ or $B$ type) to the Kleinman allowed vector or octupolar hyperpolarizabilities decreases in the anomalous dispersion regime.

\subsection{Kleinman-disallowed hyper-Rayleigh scattering}

The experimental setup of a Kleinman-disallowed hyper-Rayleigh scattering experiment (KD-HRS) is shown in Figure 1. The light from an optical parametric oscillator tunable through the near infrared is sent through a polarizer and a quarter-wave plate creating an arbitrary elliptical polarization, which is focused into a triangular quartz cell containing the chromophore in acetone solution. The scattered second harmonic light is collected at a $45^{\circ}$ angle and focused on a photomultiplier tube after a single elliptical polarization from the entire signal is selected with a similar system of a quarter-wave plate and polarizer. In the experiment, the intensity of a definite outgoing second harmonic (elliptical) is recorded as a function of the polarization of the fundamental frequency light. The essential features of this experiment that distinguishes it from a "tr aditional" HRS measurement of a Kleinman-symmetric hyperpolarizability tensor are (a) the scattering angle, which must not be 0,90 or $180^{\circ}$, and (b) the necessity to use elliptically polarized light in both the incident and scattered beams. The values of the rotational invariants of $\beta$ are extracted from the signal by means of least-square fitting. The advantage of the analytic least-square method used here is that the set of parameters (rotational invariants) that minimizes $c^{2}$ is determined analytically without the uncertainty associated with numerical gradient search methods.

Since most of our measurement wavelengths lie in the vicinity of molecular resonances, two-photon fluorescence may be a competing process that can complicate the measurements of $\beta$. To assure the dominance of the second harmonic signal over two-photon fluorescence, we measured the spectral content of the scattered light for all the chromophores at the laser excit ation wavelengths used in this study.

\subsection{Chromophores for chiral nonlinear optics}

The molecular structures of the materials studied here are shown in Figure 2. Crystal Violet (CV), Malachite Green (MG) and Brilliant Green (BG) are well-known triarylmethane dyes. Crystal Violet is often considered to have a naïve threefold symmetry. However, our measurements showed a rather strong contribution of vector components that are not allowed in non-polar point groups such as $D_{3}$ or $D_{3 \mathrm{~h}}$, and this evidence suggests deviation from these symmetries in the ground state in these solvents. These results confirm other studies that suggest that $\mathrm{CV}$ is less symmetric than expected. ${ }^{16,17}$ Unlike Crystal Violet, Malachite Green and Brilliant Green have only two out of three phenyl rings substituted with amine donors so that the resulting symmetry would be expected to be $C_{2 \mathrm{v}}$ or, given the steric repulsions $C_{2}$. Experimental results indicating devi ation of Brilliant Green from Kleinman symmetry have been previously reported. ${ }^{18}$ The two molecules synthesized in part for this study, (Compounds RT9090 and 1955-49) are $\Lambda$-shaped chromophores with the acceptor in the middle and donors on the legs of $\Lambda$. These molecules are conventional linear and dipolar NLO chromophores except that they share a common central acceptor group that results in a $\Lambda$ geometry of the overall molecule. Table 1 summarizes the results of our measurements at the excitation wavelengths of 1560,1340, 1064 and $780 \mathrm{~nm}$. The figures of merit of the two vector ( $1 \mathrm{ss}$ and $1 \mathrm{~mm}$ ), the second rank (2mm) and the third rank ( $3 \mathrm{ss}$ ) components are defined as square roots of their scalar rotational invariants (see Ref. [ 9] for precise definitions). Absolute values of invariants were found through an external referencing scheme. Para-nitroaniline ( $p$ NA) was used as a reference substance at all studied wavelengths. The value of the Kleinman-allowed vector component of

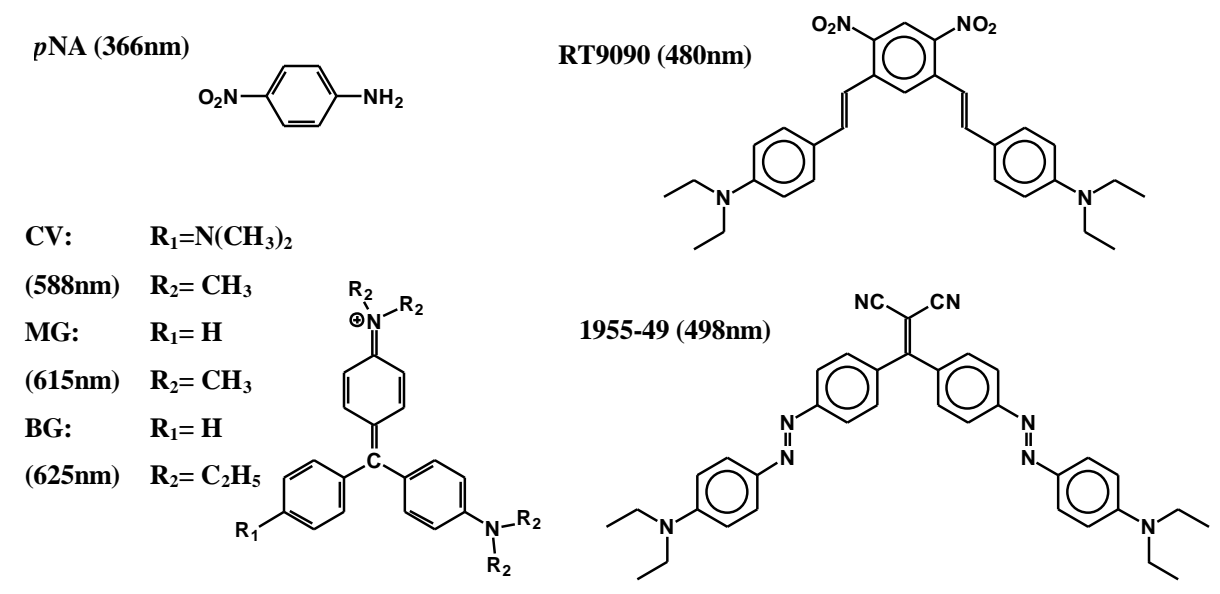

Figure 2. Molecular structures of studied materials ( with $\lambda_{\max }$ for each material in acetone solution). 
$p$ NA was derived from an EFISHG measurement at the longest wavelength ( $b_{\mathrm{EFISH}}^{\mathrm{pNA}}=12 \Delta 10^{-30} \mathrm{eSU}$ at $1580 \mathrm{~nm}$ taken from Ref. 114 of 19) and scaled for the other wavelengths accordingly to two-level dispersion model. ${ }^{20}$ One notices that all $\Lambda$ shaped molecules studied here possess sizable second rank tensor components $\left(\beta_{2 m m}\right)$ and even in the relatively off-resonance, ordinary dispersion regime at $1560 \mathrm{~nm}$ excitation, the values are comparable to the Kleinman sy mmetric ones.

\section{CHIRAL NONPOLAR ALIGNMENT FOR SECOND ORDER NLO MATERIALS}

The decomposition into irreducible parts facilitates the analysis of $\mathrm{c}^{(2)}$ as the latter is defined through rotational a verages of b. The tensor parts in Eq. (3) belong to different irreducible representation of the permutation group of three objects and thus have different permutation symmetry. After being rotationally averaged they will compose corresponding comp onents of the macroscopic tensor $\mathrm{C}$ with the same permutation symmetry that can be cla ssified in a similar fashion.

$$
\begin{aligned}
c_{I J K} \mu\left\langle b_{I J K}\right\rangle & =\left\langle b_{I J K}^{(3 s)}\right\rangle+\left\langle b_{I J K}^{(2 m)}\right\rangle+\left\langle b_{I J K}^{(1 m)}\right\rangle+\left\langle b_{I J K}^{(1 s)}\right\rangle \\
& \mu c_{I J K}^{(3 s)}+c_{I J K}^{(2 m)}+c_{I J K}^{(1 m)}+c_{I J K}^{(1 s)}
\end{aligned}
$$

Thus, each irreducible component of bulk nonlinear susceptibility c results from one component of the hyperpolarizability tensor b having the same symmetry and not the others. Consequently, it is possible to define optimization conditions for each part of $\mathrm{C}$ separately without knowing the precise form of the orientational distribution function. The knowledge of the sy $\mathrm{m}-$ metry group of the chromophore and the medium can predict that some of the four parts in Eq. (7) must vanish identically. For example, if the symmetry of the aligned medium is such that C automatically satisfies Kleinman (full permutation) sy $\mathrm{m}$ metry, the Kleinman non-symmetric parts $2 m$ and $1 m$ in Eq. (7) will have to vanish due to alignment even if the correspon ding molecular characteristics are not zero. Since the goal of this study is to achieve optimization of the NLO response through nonpolar alignment, the main focus of the further discussion will be directed at the non-vector irreducible components of $\mathrm{C}$. We are going to consider two the most important cases of chiral axial systems - uniaxially aligned media with $D_{\infty}$ symmetry,

Table 1. Figures of merit for the irreducible parts of the first hyperpolarizability tensor obtained from $45^{\circ} \mathrm{KD}-\mathrm{HRS}$ experiment. The depolarization ratio is determined from a separate $90^{\circ}$ scattering experiment and calculations from the rotational invariants. The shaded areas in

\begin{tabular}{|c|c|c|c|c|c|c|c|c|c|c|c|c|}
\hline \multirow[t]{2}{*}{ Material } & & $\left\|\beta_{1 s s}\right\|$ & & \multicolumn{2}{|l|}{$\left\|\beta_{1 \mathrm{~mm}}\right\|$} & \multicolumn{2}{|l|}{$\left\|\beta_{2 \mathrm{~mm}}\right\|$} & \multirow[t]{2}{*}{$\left\|\beta_{3 s s}\right\|$} & \multicolumn{4}{|c|}{ Depolarization Ratio } \\
\hline & & & & & esu $\times 1$ & $\left.0^{-30}\right)$ & & & $90^{\circ}$ Exper & ment & Calc. from & $45^{\circ}$ \\
\hline pNA (ref) & \multirow{4}{*}{ 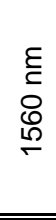 } & 7.12 & \pm 0.16 & 0.0 & \pm 2.1 & 3.0 & \pm 2.6 & $4.95 \pm 0.23$ & 0.2 & \pm 0.01 & 0.24 & \pm 0.05 \\
\hline CV & & 83.5 & \pm 2.1 & 72.2 & \pm 2.1 & 84.1 & \pm 6.5 & $76.0 \quad \pm 4.2$ & 0.63 & \pm 0.01 & 0.65 & \pm 0.06 \\
\hline MG & & 69.8 & \pm 1.8 & 14 & \pm 30 & 38.6 & \pm 9.5 & $54.0 \quad \pm 5.0$ & 0.28 & \pm 0.02 & 0.28 & \pm 0.10 \\
\hline$B G$ & & 92.7 & \pm 2.1 & 24 & \pm 24 & 57.6 & \pm 6.1 & $68.0 \quad \pm 3.2$ & 0.31 & \pm 0.02 & 0.30 & \pm 0.04 \\
\hline pNA (ref) & \multirow{3}{*}{$\begin{array}{l}\varepsilon \\
\text { c } \\
\text { 아 } \\
\stackrel{m}{0}\end{array}$} & 8.09 & \pm 0.09 & 0.0 & \pm 2.4 & 3.6 & \pm 1.1 & $5.40 \pm 0.19$ & 0.22 & \pm 0.01 & 0.23 & \pm 0.06 \\
\hline RT9090 & & 266.8 & \pm 5.5 & 15 & \pm 41 & 128.8 & \pm 4.9 & $201.4 \quad \pm 4.6$ & 0.27 & \pm 0.01 & 0.26 & \pm 0.01 \\
\hline 1955-49 & & 316.0 & \pm 9.4 & 0 & \pm 98 & 180 & \pm 35 & \pm 11 & 0.303 & \pm 0.005 & 0.30 & \pm 0.06 \\
\hline pNA (ref) & \multirow{2}{*}{$\begin{array}{l}\text { ષ્ } \\
\stackrel{0}{\circ}\end{array}$} & 11.2 & \pm 1.6 & 4.6 & \pm 1.2 & 2.1 & \pm 2.6 & $8.2 \pm 1.2$ & 0.22 & \pm 0.030 & 0.30 & \pm 0.06 \\
\hline CV & & 305 & \pm 58 & 341 & \pm 72 & 276 & \pm 52 & \pm 81 & 0.65 & \pm 0.02 & 0.67 & \pm 0.08 \\
\hline pNA (ref) & \multirow{6}{*}{\begin{tabular}{l}
$E$ \\
\multicolumn{1}{c}{} \\
$\stackrel{D}{1}$
\end{tabular}} & 56.4 & \pm 5.9 & 2 & \pm 18 & 23.4 & \pm 3.8 & 38.3 & & & 0.24 & \pm 0.05 \\
\hline CV & & 552 & \pm 42 & 353 & \pm 34 & 309 & \pm 36 & \pm 31 & 0.74 & \pm 0.02 & 0.72 & \pm 0.04 \\
\hline MG & & 122 & \pm 10 & 162 & \pm 18 & 120 & \pm 13 & \pm 14 & 1.27 & \pm 0.03 & 1.45 & \pm 0.11 \\
\hline$B G$ & & 142 & \pm 11 & 186 & \pm 16 & 136 & \pm 12 & \pm 13 & 1.40 & \pm 0.05 & 1.43 & \pm 0.03 \\
\hline & & 278 & \pm 21 & 112 & \pm 27 & 135 & \pm 19 & \pm 16 & 0.39 & \pm 0.01 & 0.40 & \pm 0.04 \\
\hline $1955-49$ & & 289 & \pm 22 & 119 & \pm 25 & 167 & \pm 16 & \pm 16 & 0.46 & \pm 0.01 & 0.46 & \pm 0.04 \\
\hline
\end{tabular}
the table indicate measur ements in the anomalous dispersion regime. 
and biaxial system with $D_{2}$ symmetry. These two types of alignment are chosen because they represent a wide range of physical systems that can be easily fabricated. The first type of alignment can be realized in stretched polymers or in uniaxial liquid crystal phases (e.g. nematic or smectic $A$ ). The second alignment scheme occurs in more ordered systems like biaxial liquid crystal phases (e.g. smectic $C$ ) or liquid-crystalline polymers where two distinct directions are defined by polymer's backbone and liquid-crystalline side-chain moieties. Clearly, due to the chirality of both symmetry groups, certain measures should be taken to assure the absence of the mirror plane symmetry in the bulk. Chirality of the bulk does not require chiral chromophores, and can be achieved by aligning achiral chromophores in a chiral fashion, as will be demonstrated below.

In general, there can be a macroscop ic hyperpolarizability coming from the $2 m$ terms only if (a) the chromophores have a symmetry such that there is at least one traceless symmetric second rank pseudo-tensor which does not change under all the allowed symmetry elements and (b) if the same is true of the macroscopic medium. In more technical grouptheoretical terms, the representation of the traceless symmetric second rank pseudotensor must contain the trivial represent ation at least once in the symmetry groups of the chromophore and of the medium.

\subsection{Uniaxial alignment of $\Lambda$-chromophores $-C_{2 v}$ chromophore and $D_{\infty}$ symmetry of the medium}

In the uniaxial alignment scheme $D_{\infty}$, the nonlinear susceptibility is fully defined by one value. In Cartesian coord inates (with $z$ being the unique axis) this is $c(D)=.c_{x y z}=c_{x y y}=-c_{y z x}=-c_{y z z}$, while all the other components vanish.

The analysis of the irreducible content of the susceptibility tensor $C$ shows that there is only the second-rank component that is non-zero in the decomposition of Eq. (7):

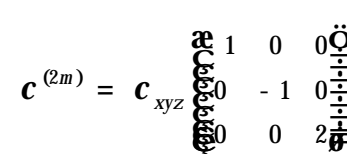

The optimization of nonlinear response of a $D_{\infty}$ medium can only be achieved by maximizing the second-rank hyperpolar izability of the chromophore and by choosing the type of alignment that is compatible with the symmetry of this component.

First, consider the more general case of an arbitrary chromophore being aligned into $D_{\infty}$ bulk. The only requirement imposed on the molecule is the existence of non-zero $b^{(2 m)}$. Using the permutation properties of $c(D$.$) , one can write it as$

$$
C_{D .}^{(2 m)}=\frac{1}{6}\left(2 C_{z z}-C_{x x}-C_{y y}\right)=\frac{1}{6} N\left\langle 2 R_{z i} R_{z j}-R_{x i} R_{x j}-R_{y i} R_{y j}\right\rangle b_{i j}^{(2 m)}
$$

Here, the hyperpolarizability given in molecular frame is transformed into laboratory coordinates with orthogonal rotation matrix $R_{i j}=\hat{i} \Delta \hat{j} \phi(\hat{i}$ and $\hat{j} \pitchfork$ stand for basis vectors of laboratory and molecular frame respectively). In writing Eq. (9) we could have chosen to calculate any non-zero combination of the tensor hyperpolarizability. The particular form that we chose above, which explicitly projects the specific traceless symmetric tensor that is non-zero in this macroscopic medium, is co nvenient, as it makes the nature of the average more evident in subsequent steps.

More specific knowledge of the chromophore's hyperpolarizability or the constraints imposed thereon by symmetry allows one to draw conclusions about alignment from Eq. (9). As it was mentioned above, $\Lambda$-shaped chromophores may exhibit large Kleinman-disallowed hyperpolarizabilities. Most $\Lambda$-shaped molecules can be approximated well as a planar (2-dimensional) $C_{2 v}$ object so that the only nonzero Cartesian components of $\mathrm{b}$ are $\mathrm{b}_{z \mathbb{z}^{\prime}} \mathrm{b}_{z x x^{\prime}} \mathrm{b}_{x z x}=\mathrm{b}_{x x z}$ (assuming $z-$ the two-fold axis and $z x$ - the plane of the molecule). The second-rank tensor part is pr esented by

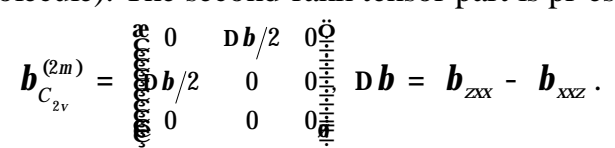

Combining Eqs. (9) and (10) together results in the expression for $b\left(C_{2 v}\right) / E c(D$.$) alignment scheme. The rot a-$ tion matrix $R$ can be defined in terms of Euler angles thus making the order parameter a function of three orientation angles of the chromophore in the bulk. The convention for Euler angles and the orientation of the molecular symmetry axis of a $\Lambda$ shaped molecule is shown in Figure 3. So, the nonlinear su sceptibility defined in Eq. (9) is now given by 


$$
\begin{aligned}
& C^{(2 m)}\left(C_{2 v} Æ E D .\right)=\frac{1}{6} N D b\left\langle 2 R_{z x} R_{z y}-R_{x x} R_{x y}-R_{y x} R_{y y}\right\rangle \\
& =\frac{1}{6} \mathrm{NDb}\langle 2(\hat{z} \nabla \hat{x} \phi(\hat{z}\langle\hat{y} \phi)-(\hat{x} \diamond \hat{x} \phi)(\hat{x} \diamond \hat{y} \phi)-(\hat{y} \Delta \hat{x} \phi(\hat{y} \Delta \hat{y} \phi) \\
& =\frac{1}{4} \mathrm{NDb}\left\langle\sin ^{2}(\mathrm{q}) \sin (2 \mathrm{y})\right\rangle
\end{aligned}
$$

The conditions for maximizing the order parameter of such a system become clear from Eq. (11). The order parameter naturally does not depend on the azimuthal angle $f$ since the system is invariant under this rotation. The macroscopic response of the system is maximized by the combination of the other two angles $q=p / 2$ and $y= \pm p / 4$. In terms of molecules' orientation, the situation corresponds to the polar axes of the molecules being perpendicular to the $C_{\infty}$-axis of the bulk and molecules being twisted about their $C_{2}$ axes by $45^{\circ}$. Note that the direction of the twist must be the same: the sign of $y$ has to be chosen either plus or minus.
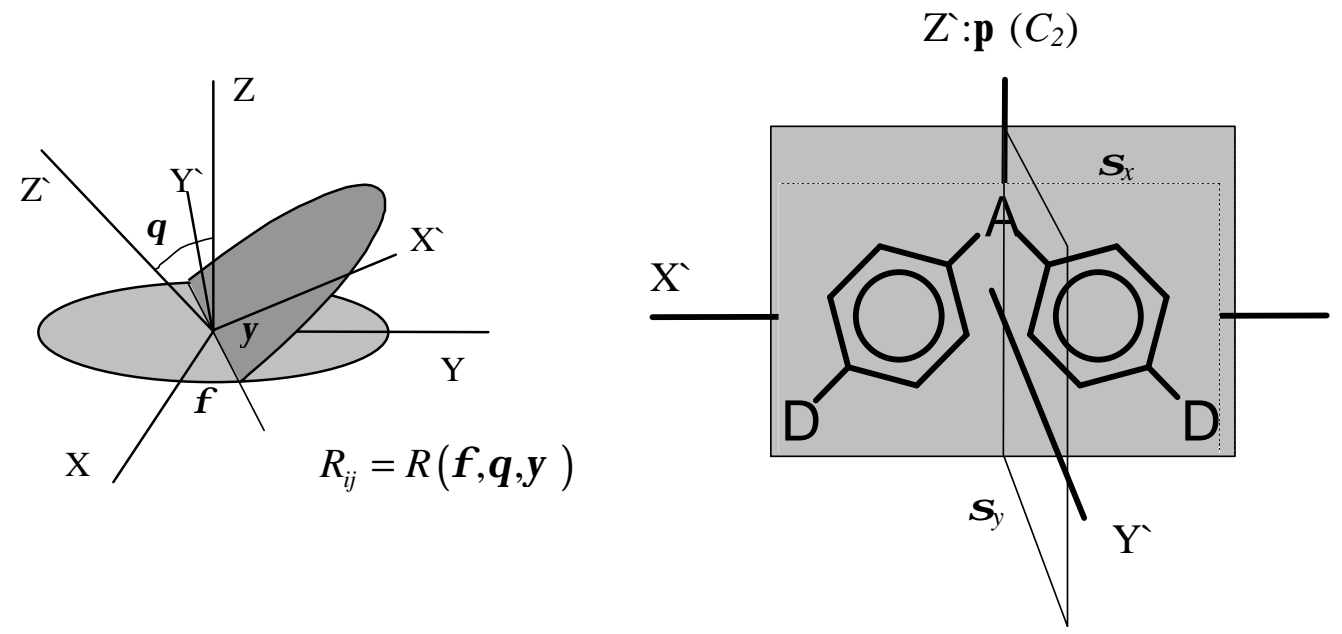

Figure 3. Euler angel rotation and molecular coordinates for a $\mathrm{L}$-shaped molecule

\subsection{Biaxial alignment of $\Lambda$-chromophores $-C_{2 v}$ chromophore and $D_{2}$ symmetry of the medium}

Consider the second chiral alignment type of interest $-D_{2}$ symmetry. The polar axis is absent in this case too so that the vector components in Eq. (7) vanish identically as in the previous case. The second and third rank tensors, however, are allowed in $D_{2}$. The tensor is defined in terms of three values (nonzero Cartesian component $\mathrm{c}_{\mathrm{xyz}}=\mathrm{c}_{x \mathrm{xy}}, \mathrm{c}_{\mathrm{yzx}}=\mathrm{c}_{\mathrm{yxz}}$, and $\mathrm{C}_{\mathrm{zxy}}=\mathrm{C}_{\mathrm{zyx}}$ ). In terms of irreducible tensors, the fully symmetric octupolar part of the susceptibility tensor $\mathrm{C}$ is defined by a single value, while the second-rank part ca rries two others

$$
\begin{array}{r}
c^{s}=c_{x y z}^{(3 s)}=c_{y z x}^{(3 s)}=\ldots=\frac{1}{3}\left(c_{x y z}+c_{y z x}+c_{z x y}\right) \\
c^{(2 m)}=\begin{array}{cccc}
\hat{K} & 0 & 0 & \hat{\tilde{z}} \\
0 & c_{z x y}-c_{x y z} & 0 & \stackrel{c_{z x y}}{\tilde{\tilde{z}}} \\
0 & 0 & c_{x y z}-c_{y z x}
\end{array}
\end{array}
$$

The second rank tensor in Eq. (12) can be split into two parts we will call uniaxial and biaxial

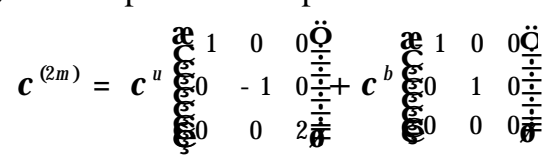



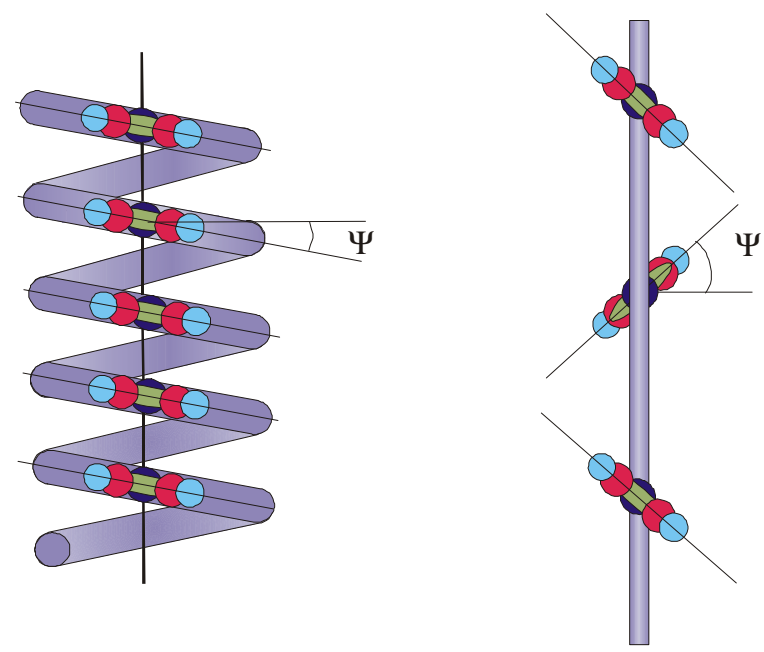

Figure 4. Example alignment of $L$-shaped molecules in a uniaxial macroscopic NLO system

$$
c^{u}=\frac{c_{x y z}-C_{y z x}}{2} ; c^{b}=\frac{2 c_{z x y}-C_{x y z}-C_{y z x}}{2}
$$

The uniaxial term of Eq. (13) is identical in structure to $C_{D \text {. }}^{(2 m)}$ (Eq. (8)) with the exception of a differently defined scalar order parameter (which, in fact, transforms into Eq. (8) upon transition of $D_{2}$ to $D_{\infty}$ ). Therefore, the entire discussion of the optimization of the macroscopic response in $D_{\infty}$ media is fully applicable to the uniaxial part of $c^{(2 \mathrm{~m})}$ so that the conditions for maximizing the order parameter $\mathrm{c}^{\mathrm{u}}$ are given by Eq. (11). The second term of Eq. (13) corresponds to biaxial alignment. The third independent value in $c\left(D_{2}\right)$ can be defined as follows

$$
c^{b}=\frac{1}{2} N\left\langle R_{y i} R_{y j}-R_{x i} R_{x j}\right\rangle b_{i j}^{(2 m)}
$$

In the special case of $C_{2 \mathrm{v}}$ chromophore (Eq. (10)) the biaxial part of the second-rank susceptibility is given by

$$
\begin{aligned}
C^{b} & =\frac{1}{2} N D b\left\langle R_{y x} R_{y y}-R_{x x} R_{x y}\right\rangle= \\
& =\frac{1}{2} N D b\langle(\hat{y} \Delta \hat{x} \phi(\hat{y} \Delta \hat{y} \phi-(\hat{x} \Delta \hat{x} \phi(\hat{x} \Delta \hat{y} \phi\rangle \\
& =\frac{1}{16} N D b\langle 4 \cos (q) \cos (2 y) \sin (2 f)+(3+\cos (2 q)) \sin (2 y) \cos (2 f)\rangle
\end{aligned}
$$

The orientational average in Eq. (15) can be maximized if the preferred polar angle of the chromophore qis close to 0 or $\pi$. Eq. (15) reduces to

$$
\begin{aligned}
& c^{b}(q=0)=\frac{1}{4} N D b\langle\sin [2(y+f)]\rangle \\
& c^{b}(q=p)=\frac{1}{4} N D b\langle\sin [2(y-f)]\rangle
\end{aligned}
$$

When the polar angle is zero, the azimuthal rotation and twist about molecule's axis become equivalent so that only the sum $y+f$ describes the orientation of the molecule. When $q=p$, the molecules are turned up side down between the first and the last rotations. The first and last rotations are also coaxial here, although positive values of $y$ correspond to negative values of $f$ and the twist angle is defined as the difference $y-f$. Therefore, the maximum of the biaxial component of the susceptibility can be achieved when the molecules are aligned along an axis pointing either up or down with the plane of the molecules twisted $45^{\circ}$ in opposite directions for upward and downward pointing species so that their molecular planes ( $x z$ - in Figure 3 ) are perpendicular. The optimized alignment schemes for $\Lambda$-shaped chromophores are summarized in Fi gure 4. 


\subsection{Propeller-like molecules $\left(D_{n}\right.$ chromophores in $D_{m}$ media, $\left.n, m>2\right)$}

Another example that we will discuss in relation to the axial alignment of Kleinman asymmetric chromophores is systems built with molecules of $D_{3}$ symmetry group that are aligned in $D_{\infty}$ fashion. Recently, various three-fold propeller-like NLO chromophores have been extensively studied in relation to their use in octupolar NLO media (e.g. Ref. 21, 22, and others). However, if the chromophore is not perfectly flat, its symmetry is compatible with the second-rank irreducible comp onent, which may be rather large as is indicated by our results for Crystal Violet ${ }^{9}$ (which, however, does not have its expected $D_{3}$ symmetry in the medium in which we have done measurements. Here we present an alignment scheme where the pe rformance of the material is based on Kleinman-disallowed $b^{(2 \mathrm{~m})}$ rather than octupolar part $b^{(3 \mathrm{~s})}$.

The symmetry group $D_{3}$ is a uniaxial group with the second-rank hyperpolarizability tensor identical by structure to Eq. (8)

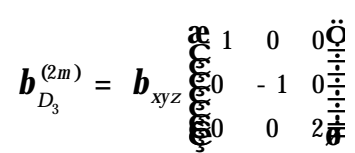

The rotational averages are even more straightforward to interpret in this case:

$$
\begin{aligned}
c\left(D_{3} \text { E E D. }\right)=C_{x y z}= & \frac{1}{6} N\left\langle 2 R_{z i} R_{z j}-R_{x i} R_{x j}-R_{y i} R_{y j}\right\rangle b_{i j}^{D_{3}} \\
& =\frac{1}{2} N b_{x y z}\left\langle 2 R_{z z} R_{z z}-R_{x z} R_{x z}-R_{y z} R_{y z}\right\rangle \\
& =\frac{1}{4} N b_{x y z}\langle 1+3 \cos (2 q)\rangle
\end{aligned}
$$

The optimal response of such a system is achieved with all molecules lying in the plane perpendicular to the sy $\mathrm{m}$ metry axis of the bulk $(q=0, p)$. The azimuthal alignment does not influence this part of the nonlinear susceptibility. The uniaxial alignment makes use of only the chiral ( $2 m$ ) part of the hyperpolarizability of a $D_{n}(n>2)$ molecule ( $\mathrm{b}_{x y z}$ ), while the utilization of the other, octupolar (3s), nonzero component $\left(b_{x x x}=-b_{x y y}=-b_{y x y}=-b_{y y x}\right)$ would require stricter, three-fold alignment that is generally harder to achieve in molecular media.

Biaxial alignment of propeller molecules is also possible. However, we know of no polymeric materials that plaus ibly have this symmetry and in consequence do not di scuss it.

\section{DISCUSSION}

The previous section discussed how chiral macroscopic NLO systems could be created via chiral alignment of chromophores that belong to a different symmetry group but contain Kleinman-disallowed (second-rank) contribution in the molecular hyperpolarizability. A set of molecular orientation angles that optimizes the macroscopic response of the material was deduced on the basis of general symmetry considerations for each case of interest. The rotational freedom of the chr omophore has to be limited although it may freely rotate in certain direction (uniaxial case). As discussed above, the alig nments described can be achieved relatively easily in polymer systems with NLO chromophores incorporated in the backbone or attached as a side chain.

A number of chiral polymeric systems for nonlinear optics have been studied recently. Van Elshocht et al. studied NLO response of Langmuir-Blodgett films made of binaphthyl-based chiral helical polymers. ${ }^{23}$ However, these systems do not seem to be optimized in the axial sense discussed here. The authors report the symmetry of the films to be $C_{\infty}$, which is supported by the observation that $C_{m \text { m }}$ is the largest component of the hyperpolarizability. Indeed, in the materials presented, the $\Lambda$-shaped monomers are attached to each other with their "feet" creating the polymer main chain, so that in the aligned state, they would rather have the planes of the molecules aligned parallel to the polymer's axis than at the $45^{\circ}$ angle - the condition that would favor large hypersusceptibility resulting from a traceless symmetric tensor hyperp olarizability.

Another work by Kauranen et al. presents a study of the NLO properties of helicenebisquinone-based LangmuirBlodgett films. ${ }^{24}$ The enantiomerically pure chiral material aggregates into a film of $C_{2}$ symmetry. However, the measurements of the nonlinear susceptibility $\chi$ showed that by far the largest Cartesian components are $C_{x y z}$ and $C_{y x z}$, i.e. the characteristic components of chiral groups $D_{\infty}$ and $D_{2}$ discussed here. So, one can imagine (at least in rough approximation) that the molecules pack in helical columns creating a structure close in symmetry to $D_{\infty}$. In this case, the chirality of the bulk is forced directly by the strong chirality of the helicenebisquinone. The molecule is essentially three-dimensional and, although clearly it is not the $C_{2 \mathrm{v}}$ case discussed in most detail above, the symmetry of the chromophore is the simpler group $C_{2}$ that has 
similar properties. The chromophore can also be thought of as a $\Lambda$-shaped chromophore. As they are stacked in columns, the molecules automatically satisfy the condition for the polar angle (pointing perpendicular to the column axis). At the same time, the "plane" of the molecule (i.e. the plane that cuts through the molecule's mid point - the "vertex" - and two end points - the "feet" of $\Lambda$ ) is naturally twisted by some (not 0 or $90^{\circ}$ ) angle away from the normal to the bulk principal axis due to the pitch of the chiral column. Moreover, the axes $x$ and $y$ discussed above in conjunction with $C_{2 \mathrm{v}}$ molecules will be rotated by some hard to discern angle which is likely to vary with frequency from (say) the plane of the central aromatic ring. Thus, given the formalism presented above, the large chiral nonlinearity reported in Ref. 24 can be accounted for by satisfactorily (if not optimally) aligned $\Lambda$-shaped molecules of helicenebisqu inone.

The desired alignment of $\Lambda$-shaped chromophores can be also achieved by other means. For a $D_{\infty}$ system, a material consisting of long, linear polymers that have $\Lambda$-shaped chromophores attached to the backbone with their vertices can be stretched in one direction, so that the molecular dipoles are, generally, perpendicular to the main chain. The linking group must contain a chiral center that favors the rotation of the plane of the chromophore by $\sim 45^{\circ}$ from the direction of the mainchain of the polymer, with the most important condition being that a preponderance of the chromophores are twisted in the same direction. An alternative scheme is somewhat similar to the system in Ref. 24: the chromophores are attached (again at their vertices) to a helical polymer backbone heading toward the helix axis. The rotation about the molecular axes must still be constrained, although it can be parallel to the local direction of the polymer chain and only tilt with respect to the symmetry axis is created (and even controlled) by pitch of the helix. The two cases are sch ematically shown in Figure 2.

The case of $D_{2}$ alignment is slightly more complicated. The structure is similar to the first example of $D_{\infty}$ alignment from the previous paragraph. The chromophores are incorporated into a linear polymer as side-chain groups with a fixed twist angle. In addition, liquid crystalline moieties are attached to the "feet" of the $\Lambda$-shaped chromophore. When conditions are satisfactory for existence of the nematic phase, the direction of the stretch (along the polymer chain) and director of the nematic phase (perpendicular to the main axis) define two distinct nonpolar axes in the system, forming $D_{2}$ symmetry. When the nematic moieties align parallel to each other the chromophores will, on average, have to point mostly "up" and "down" favoring only $q=0, p$. Such alignment can also, in principle, be achieved by stretching of an appropriately cross-linked polymer gel in a biaxial fashion.

Finally, the optimized alignment scheme for $D_{3}$ chromophores can be physically realized in a discotic nematic or columnar liquid crystals or LC-polymers. Here the chirality of such systems should originate from the chirality of the chr omophores. Hence, an enantiomerically pure chromophore should be used in for the best performance, since the left- and righthanded species, if present simultaneously, will cancel each other's hyperpolarizability, so that the hyperpolarizability is pr oportional to the enantiomeric excess. Molecules with a low threshold for the transition between left- and right-handed states (like Crystal Violet) can be stabilized if placed into a matrix (or a host liquid crystal) with a well-defined handedness, or if appropriate, optically unresponsive moieties are attached thereto.

\section{CONCLUSIONS}

In this paper, we have shown how bulk nonlinear susceptibility can be created in chiral nonpolar medium. The th eory that elucidates the criteria for optimization the nonlinear response of such chiral media has been presented. Based on the conclusions drawn from the theory, several examples of known materials as well as possible physical configurations invol ving $\Lambda$-shaped and propeller-like chromophores were discussed. This study gives guidelines for the creation of conceptually new materials for second-order nonlinear optics by implementing tensorial properties of the molecular hyperpolarizability and using an appropriate ch iral alignment scheme.

\section{ACKNOWLEDGMENTS}

The support of the National Science Foundation through the Advanced Liquid Crystalline Optical Materials (A LCOM) Science and Technology Center DMR89-20147 is gratefully a cknowledged.

\section{REFERENCES}

1. Polymers for Second-order Nonlinear Optics, G.A. Lindsay and K.D. Singer, eds. (ACS Symp. Ser. 601, Washington, D.C., 1995); Molecular Nonlinear Optics, J. Zyss, ed. (Academic, Boston, 1994).

2. J. A. Giordmaine, "Nonlinear Optical Properties of Liquids", Phys. Rev. 138, 1599-1606 (1965).

3. R. Andreu, I. Malfant, P.G. Lacroix, H. Gornitzka, and K. Nakatani, Chem. of Mater. 11, 840 (1999); N. Koroteev, V.A. Makarov, and S.N. Volkov, Opt. Commun. 157, 111 (1998); S. Wozniak and G. Wagniere, Opt. Commun. 151, 81 
(1998); F. Steybe, F. Effenberger, U. Gubler, C. Bosshard, and P. Günter, Tetrahedron 54, 8469 (1998); S. VanElschocht, T. Verbiest, M. Kauranen, A. Persoons, B.M.W. Langeveld, Voss, E.W. Meijer, J. Chem. Phys. 107, 8201 (1997); S. Van Elshocht, T. Verbiest, M. Kauranen, L. Ma, H. Cheng, K.Y. Musick, L. Pu, and A. Persoons, Chem. Phys. Lett. 309, 315 (1999).

4. D.M. Walba, D.J. Dyer, X.H. Chen, U. Muller, P. Cobben, R.F. Shao, and N.A. Clark, Mol. Cryst. Liq. Cryst. Sci. and Tech. 288, 83 (1996).

5. Y. Luo. M. Lindgren, and H. Agren, Opt. Mater. 9, 216 (1998).

6. V. Ostroverkhov, O. Ostroverkhova, R.G. Petschek, K.D. Singer, L. Sukhomlinova, R.J. Twieg, S.-X. Wang, and L.C. Chien, "Optimization of the Molecular Hyperpolarizability for Second Harmonic Generation in Chiral Media" Chem. Phys. 257, 263 (2000).

7. V. R. Thalladi, S. Brasselet, H.-C. Weiss, D. Blaster, A. K. Katz, H. L. Carrell, R. Boese, J. Zyss, A. Nangia, G. R. Desiraju, "Crystal engineering of some 2,4,6-triaryloxy-1,3,5-triazines: Octupolar nonlinear materials", J. Am. Chem. Soc., 120, 2563-2577 (1998)

8. S. F. Hubbard, R.G. Petschek, K.D. Singer, N. D'Sidocky, C. Hudson, L.C. Chien, C.C. Henderson, and P.A. Cahill, "Measurements of Kleinman-disallowed Hyperpolarizability in Conjugated Chiral Molecules", J. Opt. Soc. Am. B 15, 289-301 (1998)

9. V. Ostroverkhov, R. G. Petschek, K. D. Singer, L. Sukhomlinova, R. J. Twieg, S.-X. Wang, L. C. Chien, "Measur ements of the hyperpolarizability tensor by means of hyper-Rayleigh scattering", J. Opt. Soc. Am. B, 17, 1531-1542 (2000)

10. J. Jerphagnon, D. Chemla and R. Bonneville, Adv. in Phys. 27, 609-650 (1978).

11. D.A. Kleinman, Phys. Rev. 126, 1977 (1962).

12. See e.g. R. W. Boyd, Nonlinear Optics, Academic Press (1992).

13. J.L. Oudar and J. Zyss, Phys. Rev. A 26, 2016 (1982).

14. R. Wortmann, P. Krämer, C. Glania, S. Lebus, and N. Detzer, "Deviations from Kleinman Symm etry of the SecondOrder Polarizability Tensor in Molecules with Low-Lying Perpendicular Electronic Bands", Chem. Phys. 173, 99-108 (1992)

15. C. R. Moylan, S. Ermer, S. M. Lovejoy, I.-H. McComb, D. S. Leung, R. Wortman, P. Krdmer, and R. J. Twieg, "(Dicyanomethylene) pyrane Derivatives with $C_{2 v}$ Symmetry: An Unusual Class of Nonlinear Chromophores", J. Am. Chem. Soc., 118, 12950-12955 (1996)

16. H. B. Lueck, J. L. McHale and W. D. Edwards, "Symmetry-Breaking Solvent Effects on the Electronic Structure and Spectra of a Series of Triphenylmethane Dyes", J. Am. Chem. Soc., 114, 2342-2348 (1992).

17. Y. Maruyama, M. Ishikawa, and H. Satozono, "Femtosecond Isomerization of Crystal Violet in Alcohols", J. Am. Chem. Soc. 118, 6257-6263 (1996).

18. P. Kaatz and D. P. Shelton, "Polarized Hyper-Rayleigh Light Scattering Measurements of Nonlinear Optical Chrom ophores", J. Chem. Phys. 105, 3918-3929 (1996)

19. K.D. Singer, S.F. Hubbard, A. Schober, L. M. Hayden, and K. Johnson, "Second Harmonic Generation" in Characterization Techniques and Tabulations for Organic Nonlinear Optical Materials, M.G. Kuzyk and C.W. Dirk, eds., 311513 (Marcel Dekker, 1998)

20. J. L. Oudar, D. S. Chemla, "Hyperpolarizabilities of the nitroanilines and their relations to the excited state dipole $\mathrm{m}$ oment", J. Chem. Phys., 66, 2664- (1977).

21. M. Joffre, D. Yaron, R. J. Silbey, J. Zyss, "Second order nonlinearity in octupolar aromatic systems", J. Chem. Phys., 97, 5607-5615 (1992)

22. T. Verbiest, K. Clays, C. Samyn, J. Wolff, D Reinhoudt, A. Persoons, "Investigation of the hyperpolarizability in o rganic molecules from dipolar to octopolar systems", J. Am. Chem. Soc., 116, 9320-9323 (1994)

23. S. Van Elshocht, T. Verbiest, M. Kauranen, L. Ma, H. Cheng, K. Musick, L. Pu and A. Persoons, "Chiral 1,1'binaphthyl-based helical polymers as nonlinear optical materials," Chem. Phys. Lett. 309, 315-320 (1999)

24. M. Kauranen, S. Van Elshocht, T. Verbiest, A. Persoons, "Tensor analysis of the second-order nonlinear optical su sceptibility of chiral anisotropic thin films", J. Chem. Phys., 112, 1497-1502 (2000) 